O8 (continued)

Description: IJC is a statewide cooking and nutrition education program implemented in summer 2016 by the University of Illinois Office of Extension and Outreach. Over the course of five 2-hour lessons, youth participated in hands-on culinary skill building activities, nutrition education, recipe preparation, and healthy food tastings. Lessons took place in varied community settings, examples include summer camps, libraries, churches, community centers, and Extension offices.

Evaluation: Analyses of pre- and post-intervention surveys using paired t-tests indicated that participants experienced significant pre- to post-intervention changes in cooking self-efficacy $(\mathrm{t}(1153)=18.85$, $\mathrm{p}<.001)$, fruit and vegetable preferences $(\mathrm{t}(1132)=4.48$, $\mathrm{p}<.001)$, cooking attitudes $(\mathrm{t}(1125)=9.77, \mathrm{p}<.001)$, cooking behaviors $(\mathrm{t}(1099)=3.79, \mathrm{p}<.001)$, and self-efficacy for selecting and eating healthy foods $(\mathrm{t}(1091)=$ 7.71, $\mathrm{p}<.001$ ). Researchers also observed classes at a subset of sites and interviewed nutrition educators to assess fidelity, facilitators and barriers to program implementation, and gain insights for future curriculum modifications.

Conclusions and Implications: The IJC program resulted in significant improvements in participants' dietary attitudes and behaviors. These program effects could result in positive long-term impacts on participants' dietary health, and contribute to the evidence base supporting the efficacy of the IJC program.

Funding: Supplemental Nutrition Assistance Program Education USDA NIFA 2011-67001-30101; 4-H Foundation

\section{A Digital Approach to Behavior Change - Helping Low-Income Moms to Shop, Cook, and Eat Healthy on a Budget}

Kim Laramy, BS, klaramy@ethos-marketing.com, Ethos Marketing, 17 Ash Street, Westbrook, ME 4092

Objective: Learn how a digital approach to reaching Maine moms helped them to shop, cook, and eat healthy on a budget.

Target Audience: The campaign employed detailed targeting analysis to find and reach low-income moms and serve up easy, relevant tips and tricks.

Theory, Prior Research, Rationale: Focus group learning pointed to the fact that smartphones are low-income Moms' primary connection to the world, the way they get information and access services. Moms also shared that they want to feed their families healthy, but aren't sure how to and perceive it to be expensive.

Description: A campaign and developed an integrated approach including a content-rich, mobile-friendly website, direct mail, a digital campaign with real moms sharing their tips. Digital outreach included keyword search campaigns, digital display campaigns, YouTube videos, and content development and promotion on Facebook.
Evaluation: Independent evaluation confirmed that in one year $81 \%$ of participants reported being confident in their ability to buy healthy foods on a budget for their family, and there was a $14 \%$ increase in SNAP-Ed program participants from the previous year. The website logged average monthly impressions of over 1.5 million and logged average clicks to the website of over 3,800 per month. A Facebook campaign was launched and grew to over 2,700 fans in just over a year with a high level of engagement.

Conclusion and Implications: Social media created an engaging campaign geared directly to low-income moms. Funding: None

\section{Does Hands-On Cooking Instruction Before Adulthood Support Healthy Food Behaviors to Become Lifestyle Choices?}

Anjuman Shah, MS, Columbia University; Pam Koch, EdD, RD; Casey Wilson, MS, FamilyCook Productions; Lynn Fredericks, BA, lynn@familycookprodu ctions.com, FamilyCook Productions, 330 East 43rd Street, Suite 704, New York, NY 10017

Objective: This study examines alumni of Teen Battle Chef (TBC), a hands-on culinary program, for its impact on sustaining positive eating behaviors.

Target Audience: Qualitative interviews with 30 TBC alumni, 1-7 years post TBC, who described learning to cook in TBC and how program components supported them in making and sustaining dietary changes.

Theory, Prior Research, Rationale: TBC was designed using SCT and Social Ecological Theories. Prior research on TBC found improvements in food choices, level of physical activity, empowerment to share with others, and increased self-efficacy to prepare healthy meals.

Description of Course and Curriculum: TBC introduces culinary skills by having two teams "battle" each other in TV-like cooking demos, while exploring health benefits of each recipe. Students develop leadership qualities and skills and can move onto summer internships cooking at farmers markets, gardens, preschools, restaurants and caterers.

Evaluation: Interviews were recorded, transcribed, and coded using NVivo to identify a range of themes that propelling interviewees to change their habits. Eleven total themes ranged from adventurous eating, peer/family influence, personal weight loss, family/friends weight loss, and most influential skills acquired, to increased self-confidence/esteem and following the dietary guidelines.

Conclusions and Implications: Analysis of alumni experiences indicates strong links between development of basic culinary skill to high self confidence and self actualization. Most participants expressed satisfaction in making dietary changes, losing weight themselves, and supporting friends and family members to do the same. Participants shared their own realization that healthful food can be easy to prepare and delicious and how it became "self 
O10 (continued)

evident" that they needed to make permanent dietary changes to maintain long-term health.

Funding: None

\section{Stove to Erythrocyte: Associations} Between Fish Preparation Confidence, Fish Intake, and Omega-3 Status in Midlife Adults

Stephanie Bostic, MS, smb482@cornell.edu, Cornell University, 244 Garden Avenue, Ithaca, NY 14853; Jeffery Sobal, PhD, Cornell University

Objective: This study examined whether fish and seafood preparation confidence predicted fish and seafood intake and omega-3 blood levels.

Study Design, Setting, Participants: A cross-sectional survey was conducted in varied locations in five New York State counties. Adults age 50 to 75 were recruited. A full sample of 212 participants volunteered, and a sub-sample of 100 of those 212 provided dried blood spots (DBS). The self-report survey asked about demographics, fish and seafood intake, potential confounding variables, and several Likert-type scales, including fish and seafood preparation confidence.

Outcome Measures and Analysis: Two outcomes were assessed: total fish and seafood intake, measured by food frequency questions, and omega-3 index, analyzed using gas chromatography of DBS. Three sets of linear regressions, controlling for expected confounding variables, assessed: predictors of intake among the full sample; predictors of intake among the DBS subsample; and predictors of the omega-3 index among the DBS subsample.

Results: Higher fish and seafood preparation confidence predicted greater fish and seafood intake. An increase of one point (of five possible points) in preparation confidence scale increased intake by 1.46 ounces per week $(\mathrm{p}<0.01)$ in the full sample. Similar regression coefficients occurred for confidence and intake in the DBS subsample. Preparation confidence was not associated with the omega-3 index.

Conclusions and Implications: Fish and seafood preparation confidence may be a potential target for increasing fish and seafood consumption. Increased fish and seafood preparation confidence might be developed through experiential learning opportunities related to food provisioning by nutritionists, culinary educators, paraprofessionals, extension programs, and the food and grocery industries. Funding: Cornell University: Division of Nutritional Sciences and the Human Ecology Alumni Award Grant

\section{Impact of Exposure to Food Preparation Videos on Performance Indicators Associated with Online WIC Nutrition Education}

RobertBensley,PhD, MCHES, bensley@wmich.edu, Western Michigan University, 1903 West Michigan Avenue, Kalamazoo, MI 49087; John Brusk, MPH, Western Michigan University
Objective: Determine the impact viewing a Health eKitchen (HeK) online video had on key performance indicators (KPIs) associated with WIC online nutrition education.

Study Design, Setting, Participants, Intervention: The website, wichealth.org, is an online nutrition education program used by WIC clients in 30 states. HeK is a wichealth.org feature that provides supplemental video resources focused on food preparation, cooking, and storing. This study involved a retrospective cohort of users with groups defined based on HeK exposure before or after lesson completion.

Outcome Measures and Analysis: A one-way ANOVA compared KPIs among three groups completing a wichealth.org lesson: no videos viewed, video viewed pre-lesson, video viewed post-lesson. A two independent samples t-test between the two groups with HeK views evaluated the direct impact of video on completion of lesson.

Results: A significant group effect existed for all three KPIs investigated based on HeK exposure status. Average link views $(\mathrm{F}=1,440.9, \mathrm{p}<0.0001)$, average link view time $(\mathrm{F}=7,301.9, \mathrm{p}<0.0001)$, and average rate stage of change progressed $(\mathrm{X} 2=1,328,522.6, \mathrm{p}<0.00001)$ demonstrated more positive results for the pre-lesson $\mathrm{HeK}$ viewing group. Among the two HeK groups, those that viewed a video prior had more positive significant KPI results.

Conclusions and Implications: Accessing a $\mathrm{HeK}$ video prior to completing an online educational session had a significant impact on achieving lesson goals, suggesting the potential benefit of using an application oriented video at the onset of an online nutrition education lesson.

Funding: USDA

013 The Meaning of Food in Life Scale: Evidence for Links with Healthy Eating, a Positive Relationship to Food, and Established Determinants of Healthy Eating Behavior

Naomi Arbit, MA, MS, naomi.arbit@gmail.com, Columbia University, 525 West 120th Street, New York, NY 10027; Matthew Ruby, PhD, University of Pennsylvania;

Paul Rozin, PhD

Objective: The Meaning of Food in Life (MFL) construct was recently operationalized for empirical study and a validated MFL assessment questionnaire was created. The current study evaluated how the MFL relates to dietary behaviors, a positive relationship to food, and determinants of healthy eating behavior.

Study Design, Setting, Participants: A questionnaire was administrered to two online samples consisting of 151 and 252 American participants.

Outcome Measures and Analysis: Variables assessed included demographics, the MFL, dietary intake, self- 